\title{
Development and evaluation of a loop-mediated isothermal amplification (LAMP) assay for rapid detection of Actinobacillus pleuropneumoniae based the $d s b E$-like gene ${ }^{1}$
}

\author{
Hongwei $\mathrm{Ji}^{3}$, Haitao-Li² ${ }^{2}$ Ling Zhu³, Hui Zhang ${ }^{3}$, Yin Wang², Zhicai Zuo ${ }^{2}$, Wanzhu Guo ${ }^{3}$ \\ and Zhiwen $\mathrm{Xu}^{3 *}$
}

\begin{abstract}
Ji H.W., Li H.T., Zhu L., Zhang H., Wang Y., Zuo Z.C., Guo W.Z. \& Xu Z.W. 2012. Development and evaluation of a loop-mediated isothermal amplification (LAMP) assay for rapid detection of Actinobacillus pleuropneumoniae based the dsbE-like gene. Pesquisa Veterinária Brasileira 32(8):757-760. Key Laboratory of Animal Biotechnology, Center of Sichuan Province, College of Veterinary Medicine, Sichuan Agricultural University, Ya'an, Sichuan, 625014, P.R. China. E-mail: abtcxzw@126.com

This paper reports on the development and validation of a loop-mediated isothermal amplification assay (LAMP) for the rapid and specific detection of Actinobacillus pleuropneumoniae (A. pleuropneumoniae). A set of six primers were designed derived from the $d s b E$-like gene of A.pleuropneumoniae and validate the assay using 9 A. pleuropneumoniae reference/field strains, 132 clinical isolates and 9 other pathogens. The results indicated that positive reactions were confirmed for all $A$. pleuropneumoniae strains and specimens by LAMP at $63^{\circ} \mathrm{C}$ for $60 \mathrm{~min}$ and no cross-reactivity were observed from other non-A.pleuropneumoniae including Haemophilus parasuis, Escherichia coli, Pasteurella multocida, Bordetella bronchiseptica, Streptococcus suis, Salmonella enterica, Staphylococcus, porcine reproductive and respiratory syndrome virus (PRRSV), and Pseudorabies virus. The detection limit of the conventional PCR was $10^{2} \mathrm{CFU}$ per PCR test tube, while that of the LAMP was 5 copies per tube. Therefore, the sensitivity of LAMP was higher than that of PCR. Moreover, the LAMP assay provided a rapid yet simple test of $A$. pleuropneumoniae suitable for laboratory diagnosis and pen-side detection due to ease of operation and the requirement of only a regular water bath or heat block for the reaction.
\end{abstract}

INDEX TERMS: Actinobacillus pleuropneumoniae, loop-mediated isothermal amplification (LAMP), sensitivity.

\section{INTRODUCTION}

Actinobacillus pleuropneumoniae is a Gram-negative, encapsulated respiratory pathogen of porcine contagious pleuropneumonia. Due to the aggressive nature of $A$. pleuropneumoniae, it is distributed worldwide and outbreaks usually result in significant economic losses to the swine industry (Sebunya \& Saunders 1983). The disease is characterized by fibrinous pleuritis with hemorrhagic and necrotic lesions in the lungs (Fenwick \& Henry 1994). At

\footnotetext{
${ }^{1}$ Received on April 2, 2012.

Accepted for publication on April 27, 2012.

${ }^{2}$ College of Veterinary Medicine of Sichuan Agricultural University, Ya'an, Sichuan, 625014, P.R. China.

${ }^{3}$ Key Laboratory of Animal Biotechnology Center of Sichuan Province, College of Veterinary Medicine of Sichuan Agricultural University, Ya'an, Sichuan, 625014, P.R. China. *Corresponding author: abtcxzw@126.com
}

present, 15 different serovars and two biotypes of A. pleuropneumoniae have been described based on nicotinamide adenosine dinucleotide (NAD) requirements (Rogers et al. 1990). Serovar specificity is predominately due to structural differences in the capsular polysaccharides and for most serovars also differences in lipopolysaccharides (Chatellier et al. 1999). Recently, conventional cultivation of the bacteria from healthy carrier pigs has been improved by development of A. pleuropneumoniae-selective media (Jacobsen \& Nielsen 1995, Sebunya \& Saunders 1983). For the serological tests, such as immunomagnetic separation-based (IMS), tube agglutination test, agglutination in the presence of 2-mercaptoethanol, complement fixation test, and enzyme-linked immunosorbent assay (ELISA) has been used to detect the pathogen (Gagné et al. 1998, Gottschalk et al. 1994, 1994b, Gottschalk et al. 1997, Catia \& Itamar 2003). Detection of the bacterium by polymerase chain reaction 
(PCR) amplification has also been reported (Chiers et al. 2001, Gram \& Ahrens 1998, Moral et al. 1999, Schaller et al. 2001). Among these diagnostic methods, polymerase chain reaction methods are the most rapid and able to detect a small amount of bacterial chromosomes. Although the PCR assay have been shown to be highly effective for A. pleuropneumoniae detection, the requirement of an expensive specialist thermal cycler limit their universal application, particularly in resource-limited or point-of-care settings and clinical laboratories.

Loop-mediated isothermal amplification (LAMP) is a novel nucleic acid amplification method (Nagamine et al. 2001,2002 , Notomi et al. 2000). The method is characterized by employing a DNA polymerase with strand-displacement activity, along with two inner primers (FIP, BIP) and two outer primers $(\mathrm{F} 3, \mathrm{~B} 3)$ to form auto-cycling immediates. The new assay is a novel technique which is quite simple, requiring only a conventional water bath or heat block for incubation under isothermal conditions. The sensitivity, specificity, and applicability of the method have been reported. Because of these advantages, it has been applied successfully to the detection of a lot of pathogen, such as Newcastle disease virus, Escherichia coli, PRRSV (Hong et al. 2004, Song et al. 2005, Chen et al. 2008).

\section{MATERIALS AND METHODS}

\section{Bacterial strains.}

All strains (China Institute of Veterinary Drugs Control) used for this study were listed in Table 1. Actinobacillus pleuropneumoniae serotype 3 was used to develop a LAMP method and determine the detection limit of the method. A. pleuropneumoniae were grown overnight in tryptic soy broth (TSB) medium at $37^{\circ} \mathrm{C}$ shaking on a rotary shaker, supplemented with $10 \mu \mathrm{g}$ of $\mathrm{NAD} / \mathrm{ml}$ and $10 \%$ bovine serum. Bordetella bronchiseptica was grown on Bordet-Gengou agar supplemented with $10 \%$ sheep's blood. All bacterium/virus were identified by conventional PCR (or RT-PCR) and sequencing.

\section{DNA/RNA extraction}

DNA from A. pleuropneumoniae and other species were prepared as follows: bacterial cells of each strain from colonies on TSA were resuspended in TE buffer [10mM Tris-HCl(pH 8.0), $1 \mathrm{mM}$ EDTA] to achieve a concentration of approximately $10^{6} \mathrm{CFUmL}^{-1}$. All the strains were treated in a boiling water bath for $10 \mathrm{~min}$ and centrifuged for $10 \mathrm{~min}$. The resulting supernatant was used as the template for the LAMP. For PRRSV and Pseudorabies virus, DNA and RNA were extracted directly from virus with a Trizol reagent (Invitrogen), according to the manufacturer's instructions. Complementary DNA (cDNA) synthesis reaction was performed by the TranScript First-strand cDNA Synthesis SuperMix (Beijing TransGen Biotech Co., China) in accordance with the manufacturer's instructions.

\section{Primer design and LAMP reaction}

A set of 6 primers for LAMP were designed, using the online LAMP PRIMER DESIGN software (https://primerexplorer. jp/e/),by targeting highly conserved and $A$.pleuropneumoniae specific sequences regions of $d s b E$-like gene (Chiers et al. 2001). Primer names and sequences for LAMP are shown in Table 2. The LAMP reaction was carried out in a conventional water bath, the total $25-\mu \mathrm{L}$ reaction mixture containing $2.0 \mu \mathrm{M}$ each of FIP and BIP
Table 1. Strains used test species-specific of the LAMP for detection of Actinobacillus pleuropneumoniae

\begin{tabular}{lcccc}
\hline \multicolumn{1}{c}{ Strains result } & $\begin{array}{c}\text { Reference/ } \\
\text { Field strain }\end{array}$ & Serotype & $\begin{array}{c}\text { Number of } \\
\text { strains tested }\end{array}$ & LAMP \\
\hline A. pleuropneumoniae & Reference & 1 & 1 & $1 / 1$ \\
& Reference & 2 & 1 & $1 / 1$ \\
& Reference & 3 & 1 & $1 / 1$ \\
& Reference & $5 \mathrm{a}$ & 3 & $3 / 3$ \\
& Reference & $5 \mathrm{~b}$ & 1 & $1 / 1$ \\
& Reference & 7 & 1 & $1 / 1$ \\
& Field & 8 & 2 & $2 / 2$ \\
& Field & 9 & 1 & $1 / 1$ \\
Haemophilus parasuis & Field & 12 & 1 & $1 / 1$ \\
Escherichia coli & Field & & 1 & $0 / 1$ \\
Pasteurella multocida & Reference & & 3 & $0 / 3$ \\
Bordetella bronchiseptica & Field & & 8 & $0 / 8$ \\
Streptococcus suis & Field & & 4 & $0 / 4$ \\
Salmonella enterica & Field & & 3 & $0 / 3$ \\
Staphylococcus & Reference & & 4 & $0 / 4$ \\
PRRSV & Field & & 1 & $0 / 1$ \\
Pseudorabies virus & Field & & 6 & $0 / 6$ \\
& Field & & 5 & $0 / 5$
\end{tabular}

Table 2. Details of LAMP and PCR primers designed for detection of Actinobacillus pleuropneumoniae

\begin{tabular}{ccl}
\hline Method & Primer & \multicolumn{1}{c}{ Sequence } \\
\hline \multirow{2}{*}{ LAMP } & F3 & 5'-CTGAAAGTTTCGTCAGCAC-3' \\
& B3 & 5'-TCTGAAACTGAAAAGTATCCAC-3' \\
& FIP (F1+F2) & 5'-GCTCGAAATCCGGGTTATATCCT- \\
& & TTTT-ATTTAATTGATAAACACGGTGTG-3' \\
& BIP(B1+B2) & 5'-TCGTTTAAAAGCGTTACAAGAGGA- \\
PCR & F & 'TTT-AAACTGAAGACAAGCGGTA-3' \\
& B & 5'-GACAAACCTTTTCCGGAATT-3' \\
& &
\end{tabular}

primer, $0.2 \mu \mathrm{M}$ each of F3 and B3 primer, $1.4 \mu \mathrm{M}$ each deoxynucleoside triphosphate, $8 \mathrm{U}$ of Bst DNA polymerase (New England Biolabs) using the manufacturer's supplied $10 \times$ buffer (containing $2 \mathrm{mM}$ of MgSO4, $0.8 \mathrm{M}$ betaine) and $1 \mu \mathrm{l}$ of extracted template DNA in a $0.2 \mathrm{ml}$ Eppendorf tube. Before the reaction, to increase the sensitivity of the LAMP, the reaction mixture (without the Bst DNA polymerase) was heated at $96^{\circ} \mathrm{C}$ for $5 \mathrm{~min}$ and immediately cooled on ice for $3 \mathrm{~min}$. The amplification reaction was performed at $63^{\circ} \mathrm{C}$ for $60 \mathrm{~min}$ and then terminated by heating at $95^{\circ} \mathrm{C}$ for 5 min. Each test was repeated three times.

\section{Specificity and sensitivity of LAMP for Actinobacillus pleurop- neumoniae}

To evaluate the species specificity of the LAMP test, 35 strains representing 9 species (Table 1), normally found in pigs, were examined. Serial dilutions of $1,5,10,10^{2}, 10^{3}, 10^{4}$, and $10^{5}$ copies of DNA from A. pleuropneumoniae were used as template for LAMP to detection limit of the method. The sensitivity of detection was compared between LAMP and PCR (Chiers et al. 2001).

\section{Evaluation of LAMP with clinical samples}

For further evaluation of the LAMP assay, 132 samples of tonsil tissues $(n=132)$, all originating from different herds, were obtained from pigs with an apparent infection of the respiratory tract. 35 tonsil samples $(n=35)$ taken from 35 healthy pigs that was free of infection with A. pleuropneumoniae. Approximately total of $0.5 \mathrm{~g}$ of each tonsils described above was reduced to small pieces with a scalpel and placed in sterile tubes added $5 \mathrm{~mL}$ of trypticase soy broth, $5 \mathrm{~mL}$ NAD and $500 \mathrm{~mL}$ sterilized fetal bovine serum and Bacterial growth was harvested after grown overnight 
at $37^{\circ} \mathrm{C}$. Take out $2 \mu \mathrm{l}$ of each sample for LAMP and PCR ${ }^{4}$. The IMS bacterial isolation detection of $A$. pleuropneumoniae was performed according to the described previously (Gagne et al. 1998).

\section{RESULTS AND DISCUSSION}

The analysis on agarose gel $2.5 \%$ indicated an amplification successful by LAMP showed a ladder-like pattern appeared when using the Actinobacillus pleuropneumoniae as a template, whereas other bacterial was not amplified (Fig.1). The 9 A.pleuropneumoniae reference/field strains could all get the positive reaction, indicated that these LAMP assay could detect each of the A. pleuropneumoniae given in these LAMP method (Fig.2). In order to rule out the possibility of false positivity, all the positive products of LAMP were sequenced and the results were as expected. The sensitivity of LAMP was demonstrated by using various A. pleuropneumoniae DNA dilutions as templates. As shown in Figure

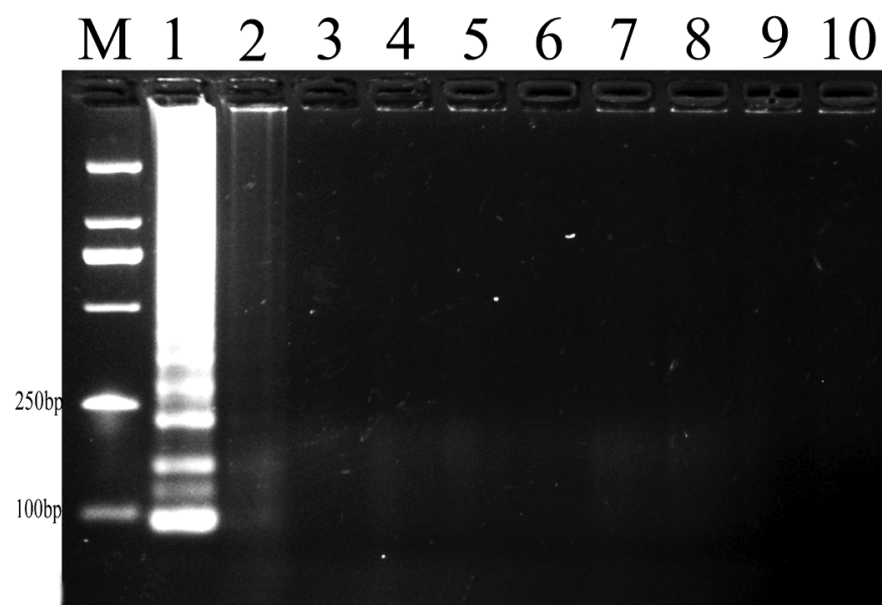

Fig.1. The specificity of LAMP assay for detecting Actinobacillus pleuropneumoniae. Amplification was detected by electrophoretic analysis on a 2.5\% agarose gel. Lane M, DNA marker DL2000, lane 1 A. pleuropneumoniae; lane 2-10: Haemophilus parasuis, Escherichia coli, Pasteurella multocida, Bordetella Bronchiseptica, Streptococcus suis, Salmonella enterica, Staph ephylococcus, PRRSV, Pseudorabies virus.

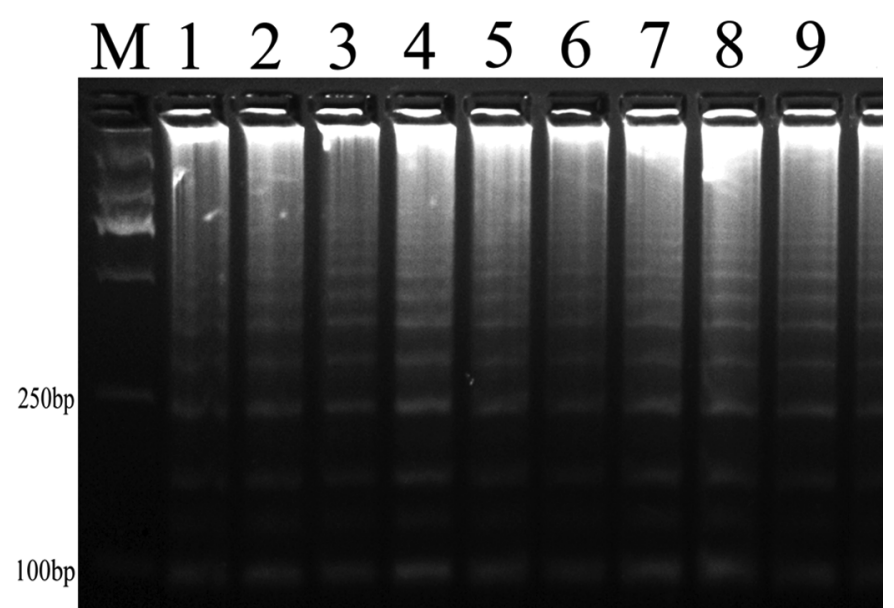

Fig.2. The LAMP assay for detecting 9 A pleuropneumoniae reference/field strains. Lane M, DNA marker DL2000, lanes 1-9: Serotype 1, Serotype 2, Serotype 3, Serotype 5a, Serotype 5b, Serotype 7, Serotype 8, Serotype 9, Serotype 12 , respectively.
3 , electrophoresis results revealed that it was possible to detect 5 copies. However, as previously reported, the PCR assay could detect the DNA was $10^{2} \mathrm{CFU}$ per PCR test tube (Chiers et al. 2001). Therefore, the LAMP method was more sensitive than the PCR described previously The detection sensitivity of LAMP was 20 -fold higher than the PCR described previously

The feasibility of the LAMP assay for detecting A. pleuropneumoniae in clinical material was assessed by using healthy pigs and infected pigs. Among the 35 samples collected from healthy pig, there was no false positivity for the three assays. For the sensitivity, the LAMP gave a total of 73 positive results and 19 samples were positive for LAMP but negative for the PCR method (Table 3). The positive results obtained by IMS bacterial isolation is 38 , and these positive results also tested positive by both PCR and LAMP. Overall, the LAMP method demonstrated higher sensitivity than that of PCR and IMS bacterial isolation.

Table 3. Comparison the sensitivity of the conventional PCR, IMS bacterial isolationand LAMP for detection of Actinobacillus pleuropneumoniae

\begin{tabular}{lcc}
\hline \multirow{1}{*}{ Assay } & \multicolumn{2}{c}{ Positive samples (\%) } \\
\cline { 2 - 3 } & Healthy material & infected material \\
\hline LAMP & $0 \%(0 / 35)$ & $53 \%(73 / 132)$ \\
PCR & $0 \%(0 / 35)$ & $41 \%(54 / 132)$ \\
IMS bacte-rial isolation & $0 \%(0 / 35)$ & $29 \%(38 / 132)$
\end{tabular}

Porcine contagious pleuropneumonia is highly contagious and may result in high herd mortality. Pigs from 2 to 6 months are particularly affected, even though all ages are sensitive to the pathogen. Depending on the immune status of animals, environmental conditions, infecting dose and strain virulence, pigs can develop a peracute, acute, subacute or chronic form of the disease. These infected pigs can transmit A. pleuropneumoniae to the other pigs of the herd. In this context, early detection of this bacterium is important for control and treatment of the disease.

Loop-mediated isothermal amplification (LAMP) is a novel amplification method which was developed originally by Notomi (Notomi et al. 2000). This simple and rapid method relies on strand-displacing DNA synthesis performed using the large fragment of $B s t$ DNA polymerase under isothermal conditions at $60-65^{\circ} \mathrm{C}$ within $60 \mathrm{~min}$. A novel system for the rapid and easy identification of A. pleuropneumoniae was described. Another useful feature of LAMP is that its products can be observed directly by naked eye, because a white precipitate of magnesium pyrophosphate forms in the reaction tube (Mori et al. 2001).

A novel system for the rapid and easy identification of $A$. pleuropneumoniae was described. Compared to PCR, use of LAMP to detect the pathogen has many advantages. Sensitivity, it requires only small amounts of target DNA to be present in a sample for positive diagnosis, the detection limits of the LAMP described in this study was 5 copies, but that obtained by previously reported PCR was $10^{2}$ CFU per PCR test tube. Moreover, in the field trial, $53 \%$ and $41 \%$ samples tested positive by LAMP and PCR, respectively. Time, it will take $60 \mathrm{~min}$ for LAMP to detect the A. pleuropneu- 
moniae, whereas the PCR method typically requires $2-4 \mathrm{~h}$. Requirements for specialist equipment, the method can be performed under isothermal conditions in the temperature range of $60-65^{\circ} \mathrm{C}$ using a simple heating device such as a water bath or heat block, but for PCR, a complicated thermal cyclers was need. In conclusion, although PCR assays possess many advantages, the LAMP assay showed superior sensitivity than the PCR for detection of A. pleuropneumoniae and the LAMP assays can potentially be used for tests in the field with specificity improved instruments was not required and great sensitivity results can be received.

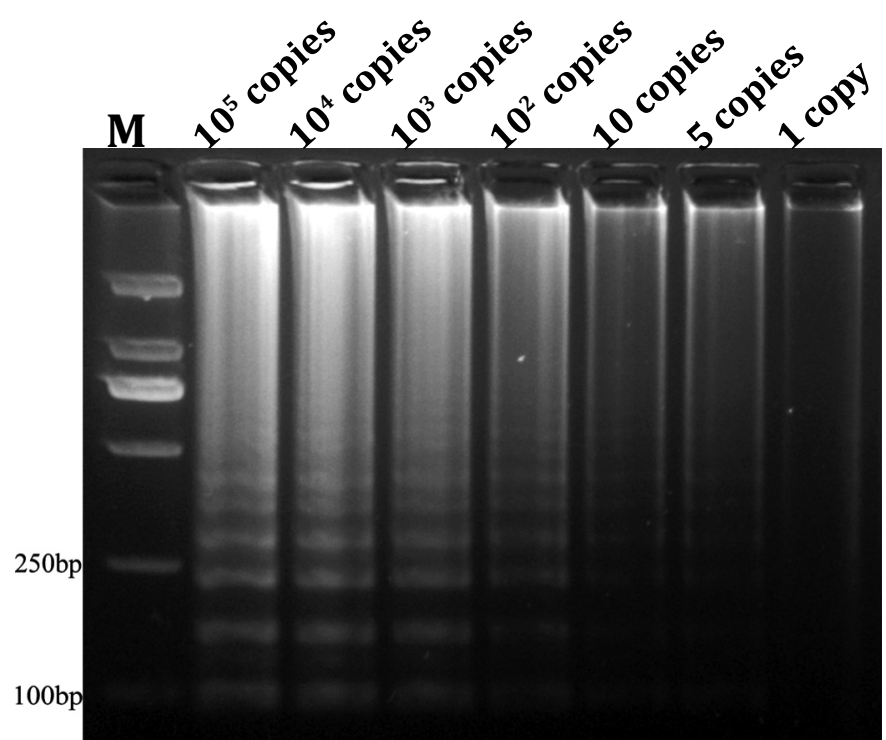

Fig.3. The detection limits of LAMP for the detection of A. pleuropneumoniae. Lane M, DNA marker DL2000, other lanes, as showed in the picture, represented the concentrations of DNA from APP as a template for LAMP.

\section{CONCLUSION}

In conclusion, a novel system for the rapid and easy identification of Actinobacillus pleuropneumoniae was described which was rapid, highly sensitive, simple, and inexpensive. This method can potentially be used for tests in the field.

\section{REFERENCES}

Catia S.K. \& Itamar A.P. 2003. Detection of Actinobacillus pleuropneumoniae by PCR on field strains from healthy and diseased pigs. Curr. Microbiol. 46:443-447.

Chatellier S., Harel J., Dugourd D., Chevallier B., Kobisch M. \& Gottschalk M. 1999. Genomic relatedness among Actinobacillus pleuropneumoniae field strains of serotypes 1 and 5 isolated from healthy and diseased pigs. Can. J. Vet. Res 63:170-176.

Chen H.T., Zhang J., Sun D.H., Ma L.N., Liu X.T., Quan K. \& Liu Y.S. 2008. Reverse transcription loop-mediated isothermal amplification for the detection of highly pathogenic porcine reproductive and respiratory syndrome virus. J. Virol. Methods 153:266-268.

Chiers K., Van I., Donné E., Baele M., Ducatelle R., De B.T. \& Haesebrouck
F. 2001. Detection of Actinobacillus pleuropneumoniae in cultures from nasal and tonsillar swabs of pigs by a PCR assay based on the nucleotide sequence of a $d s b E$-like gene. Vet. Microbiol. 83:147-159.

Fenwick B.W. \& Henry S. 1994. Porcine pleuropneumonia. J. Am. Vet. Med. Assoc. 204:1334-1340.

Gagné A., Lacouture S., Broes A., D’Allaire S. \& Gottschalk M. 1998. Development of an immunomagnetic method for selective isolation of Actinobacillus pleuropneumoniae serotype 1 from tonsils. J. Clin. Microbiol. 36:251-254

Gottschalk M., Altman E., Charland N., De L.F. \& Dubreuil J.D. 1994. Evaluation of a saline boiled extract, capsular polysaccharides and long-chain lipopolysaccharides of Actinobacillus pleuropneumoniae serotype 1 as antigens for the serodiagnosis of swine pleuropneumonia. Vet. Microbiol. 42:91-104.

Gottschalk M., Altman E., Charland N., De L.F. \& Dubreuil J.D. 1997. Serodiagnosis of swine pleuropneumonia due to Actinobacillus pleuropneumoniae serotypes 7 and 4 using long-chain lipopolysaccharides. Can. J. Vet. Res. 61:62-65.

Gottschalk M., De L.F., Radacovici S. \& Dubreuil J.D. 1994b. Evaluation of long chain lipopolysaccharides (LC-LPS) of Actinobacillus pleuropneumoniae serotype 5 for the serodiagnosis of swine pleuropneumonia. Vet. Microbiol. 38:315-327.

Gram T. \& Ahrens P. 1998. Improved diagnostic PCR assay for Actinobacillus pleuropneumoniae based on the nucleotide sequence of an outer membrane lipoprotein. J. Clin. Microbiol. 36:443-448.

Hong T.C., Mai Q.L., Cuong D.V., Parida M., Minekawa H., Notomi T., Hasebe F. \& Morita K. 2004. Development and evaluation of a novel loop-mediated isothermal amplification method for rapid detection of severe acute respiratory syndrome coronavirus. J. Clin. Microbiol. 43:1956-1961.

Jacobsen M.J. \& Nielsen J.P. 1995. Development and evaluation of a selective and indicative medium for isolation of Actinobacillus pleuropneu-moniae. Vet. Microbiol. 47:191-197.

Moral C.H., Soriano A.C., Salazar M.S. \& Marcos J.Y. 1999. Molecular cloning and sequencing of the aroA gene fromActinobacillus pleuropneumoniae and its use in a PCR assay for rapid identification. J. Clin. Microbiol. 37:1575-1578.

Mori Y., Nagamine K., Tomita N. \& Notomi T. 2001. Detection of loop-mediated isothermal amplification reaction by turbidity derived from magnesium pyrophosphate formation. Biochem. Biophys. Res. Commun. 289:150-154.

Nagamine K., Kuzuhara Y. \& Notomi T. 2002. Isolation of single-stranded DNA from loop-mediated isothermal amplification products. Biochem. Biophys. Res. Commun. 290:1195-1198.

Nagamine K., Watanabe K., Ohtsuka K. \& Hase T. 2001. Loop-mediated isothermal amplification reaction using a nondenatured template. Clin. Chem. 47:1742-1743.

Notomi T., Okayama H., Masubuchi H. \& Yonekawa T. 2000. Loop-mediated isothermal amplification of DNA. Nucleic Acids Res. 28:E63.

Rogers R.J., Eaves L.E., Blackall P.J. \& Truman K.F. 1990. The comparative pathogenicity of four serovars of Actinobacillus (Haemophilus) pleuro-pneumoniae. Aust. Vet. J. 67(1):9-12.

Schaller A., Djordjevic S.P., Eamens G.J. \& Forbes W.A. 2001. Identification and detection of Actinobacillus pleuropneumoniae by PCR based on the gene apxIVA. Vet. Microbiol. 79:47-62.

Sebunya T.N.K. \& Saunders J.R. 1983. Haemophilus pleuropneumoniae infection in swine: A review. J. Am. Vet. Med. Assoc. 182:1331-1337.

Song T., Toma C., Nakasone N. \& Iwanaga M. 2005. Sensitive and rapid detection of Shigella and enteroinvasive Escherichia coli by a loop-mediated isothermal amplification method. FEMS Microbiol. Lett. 243:259263. 